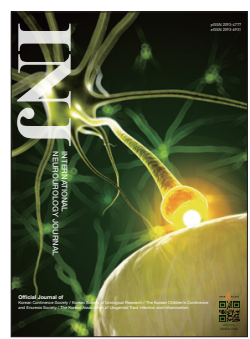

\title{
Association Study of Polymorphisms of Epidermal Growth Factor and Epidermal Growth Factor Receptor With Benign Prostatic Hyperplasia in a Korean Population
}

\author{
Su Kang Kim ${ }^{1, \star}$, Hyun Kyung Park ${ }^{2, *}$, Han Sung Choi ${ }^{2}$, Koo Han Yoo ${ }^{3}$, Joo-Ho Chung ${ }^{1}$ \\ ${ }^{1}$ Kohwang Medical Research Institute, Kyung Hee University School of Medicine, Seoul, Korea \\ ${ }^{2}$ Department of Emergency Medicine, Kyung Hee University School of Medicine, Seoul, Korea \\ ${ }^{3}$ Department of Urology, Kyung Hee University School of Medicine, Seoul, Korea
}

Purpose: Recent studies have suggested that specific single-nucleotide polymorphisms (SNPs) contribute to the clinical features of benign prostatic hyperplasia $(\mathrm{BPH})$. In this study, we investigated the relationships of genetic polymorphisms of the epidermal growth factor $(E G F)$ gene and the epidermal growth factor receptor (EGFR) gene with BPH.

Methods: A total of 218 patients with BPH were enrolled in this study. We evaluated the relationship between eight SNPs in the EGF and EGFR genes and prostate volume, prostate-specific antigen (PSA), and International Prostate Symptom Score of $\mathrm{BPH}$ patients. Each SNP was genotyped by direct sequencing. Statistical analysis applying codominant, dominant, recessive, and $\log$-additive models was performed via logistic regression.

Results: The rs11568943 and rs11569017 SNPs in the EGF gene showed significant associations with prostate volume (rs11568943: $\mathrm{P}=0.038$ in the log-additive model, $\mathrm{P}=0.024$ in the allele distribution; rs11569017, $\mathrm{P}=0.031$ in the dominant model, $\mathrm{P}=0.028$ in the log-additive model, $\mathrm{P}=0.020$ in the allele distribution). Additionally, the rs3756261, rs 11568943 , and rs 11569017 SNPs of the EGF gene and the rs2293347 SNP of the EGFR gene were associated with PSA levels $(\mathrm{P}<0.05$ in each model, respectively).

Conclusions: These results suggest that the EGF gene may affect prostate volume. In addition, the EGF and EGFR genes may be associated with PSA levels in patients with BPH.

Keywords: Epidermal Growth Factor; Epidermal Growth Factor Receptor; Polymorphism, Genetic; Polymorphism, Single Nucleotide; Prostatic Hyperplasia

- Research Ethics: This study was approved by Institutional Review Board of Kyung Hee University Medical Center in 2009 (KMC IRB 0913-03).

- Conflict of Interest: No potential conflict of interest relevant to this article was reported.

\section{INTRODUCTION}

Benign prostatic hyperplasia (BPH) is a pathologic process that is one of the causes of lower urinary tract symptoms (LUTS) in aging men. BPH may cause symptoms including LUTS, benign prostatic enlargement, and bladder outlet obstruction. BPH has similar risk factors to those of prostate cancer [1]. Epithelial cell proliferation in prostate tissue is a major pathologic feature of
Corresponding author: Joo-Ho Chung (iD http://orcid.org/0000-0003-3517-7175 Kohwang Medical Research Institute, Kyung Hee University School of Medicine, 26 Kyungheedae-ro, Dongdaemun-gu, Seoul 02447, Korea

E-mail: jhchung@khu.ac.kr / Tel: +82-2-961-0281 / Fax: +82-2-968-0560

*These authors contributed equally to this study and should be considered co-first authors.

Submitted: February 16, 2016 / Accepted after revision: August 12, 2016 
$\mathrm{BPH}$ [2]. Epidermal growth factor (EGF) is linked to the growth and differentiation of epithelial cells. The relationship between EGF and prostate cancer has been previously studied. EGF, vascular endothelial growth factor, fibroblast growth factor 2 , transforming growth factor beta 1 , and insulin-like growth factor 1 are known to influence the development of both prostate cancer and $\mathrm{BPH}[3,4]$, and both conditions share risk factors. However, the relationship between BPH and EGF remains unclear [1].

Evidence has emerged suggesting that the action of EGF is involved in prostate cell growth. EGF expression is promoted by exposure to androgens, such as testosterone and dihydrotestosterone [5-7]. EGF may cause elevations in E-cadherin, which may promote the phosphorylation of epidermal growth factor receptor (EGFR) and cell proliferation signals [8]. Recent studies have shown that the inhibition of EGFR was linked to decreased proliferation signals to prostatic epithelial cell lines [9].

Although several studies have investigated EGF, only 1 polymorphism in the EGF gene has been reported to affect the development of prostate cancer. Teixeira et al. [10,11] reported that the $E G F+61 \mathrm{G}>$ A polymorphism may contribute to prostate cancer susceptibility and androgen insensitivity. Studies of the EGFR gene have shown the features or development of prostate cancer to be associated with various exon mutations $[12,13]$ and single-nucleotide polymorphisms (SNPs) including rs17172432 [14], rs6964705 in the EGFR gene combined with rs1401862 in the matrix metallopeptidase 16 (MMP16) gene [15], and rs884419 [16].

Although the above studies support a relationship between EGF signals and proliferative prostate disease, no study has investigated the relationship of $\mathrm{BPH}$ with the EGF and EGFR genes. In the present study, we investigated the relationship between SNPs of the EGF and EGFR genes and the clinical features of $\mathrm{BPH}$ in a Korean population.

\section{MATERIALS AND METHODS}

\section{Study Subjects}

All subjects were recruited from the Kyung Hee University Medical Center of Kyung Hee University in Seoul, Korea. This study was approved by Institutional Review Board of Kyung Hee University Medical Center in 2009 (KMC IRB 0913-03). A total of $218 \mathrm{BPH}$ patients diagnosed by a physician were selected (Table 1). The criteria for diagnosing BPH included prostate weight ( $>20 \mathrm{~g}$ ) and LUTS. The prostate-specific antigen (PSA)
Table 1. Demographic and biochemical characteristics of the benign prostatic hyperplasia patients

\begin{tabular}{lc}
\hline Characteristic & Value \\
\hline No. of subject & 218 \\
Age $(\mathrm{yr})$ & $65.6 \pm 10.3$ \\
Prostate volume $(\mathrm{mL})$ & $39.2 \pm 21.7$ \\
$<30$ & $97(44.5)$ \\
$\geq 30$ & $121(55.5)$ \\
PSA (ng/mL) & $4.6 \pm 5.4$ \\
$<1.5$ & $75 / 217(34.6)$ \\
$\geq 1.5$ & $142 / 217(65.4)$ \\
IPSS & $17.3 \pm 7.9$ \\
$0-19$ & $123 / 200(61.5)$ \\
$\geq 20$ & $77 / 200(38.5)$ \\
\hline
\end{tabular}

Values are presented as mean standard error or number (\%).

PSA, prostate-specific antigen; IPSS, International Prostate Symptom Score.

level in the serum of BPH patients was tested and prostate volume was measured using transrectal ultrasonography by urologists. LUTS were quantified using the International Prostate Symptom Score (IPSS). The subjects were dichotomized according to the criteria used in several multicenter studies: low $(0-19)$ and high $(\geq 20)$ IPSS score, low $(<1.5 \mathrm{ng} / \mathrm{mL})$ and high $(\geq 1.5 \mathrm{ng} / \mathrm{mL})$ PSA level, and small $(<30 \mathrm{~mL})$ and large $(\geq 30$ $\mathrm{mL}$ ) prostate volume. Voiding symptoms were classified using the IPSS score as mild (0-7), moderate (8-19), or severe (20-35).

The exclusion criteria were prostate cancer, neurogenic bladder, urinary tract infection, uncontrolled diabetes mellitus, and cardiovascular disease.

Written informed consent was obtained from all participants. Blood samples were collected in tubes containing ethylenediaminetetraacetic acid as an anticlotting factor and stored at $-20^{\circ} \mathrm{C}$ until use. Genomic DNA was extracted using a blood extraction kit (Roche, Indianapolis, IN, USA).

\section{SNP Selection and Genotyping}

The National Center for Biotechnology Information SNP database was searched to select SNPs of the EGF and EGFR genes for study (http://www.ncbi.nlm.nih.gov/SNP, BUILD 141). The criteria for the selection of exonic SNPs and promoter SNPs in each gene were the following: (1) > 10\% minor allele frequency, (2) $>0.1$ heterozygosity, (3) known genotype frequencies in the Asian population, and (4) previous studies. We ultimately selected 5 SNPs of the EGF gene (rs3756261, -1744 A/G; rs11568835, -1380 G/A; rs11568943, Arg431Lys; rs2237051, Met708Ile; and 
Table 2. Sequences for PCR

\begin{tabular}{lllc}
\hline SNPs & \multicolumn{1}{c}{ Sense primer } & \multicolumn{1}{c}{ Antisense primer } & PCR product size (bp) \\
\hline EGF & & & 360 \\
rs3756261 & CGCCTGGCTAACTTTTTGTATT & TTACATGTCACCTGGGCTAATG & 329 \\
rs11568835 & ATCCAAACAGAACAGAGCTGTG & GCTCTGAACCCTTACAGGAGAA & 364 \\
rs11568943 & CACAGTGGCTCACACCTGTAAT & GGAAAATCAATTCTTCCTTGAC & 351 \\
rs2237051 & AGTCGGTGGCTCACTCATAACT & CAGCCAAGGAAAGACTGTGTAA & 446 \\
rs11569017 & CATCTTCAAACCCACTTGTGAA & CACTATAAATGGGGAGGTGGAG & \\
EGFR & & & 352 \\
rs6965469 & GTTCAGCAAACCCATTCTTCTC & CCTGTGCATTCACTTAACAAGG & 323 \\
rs2293347 & AACAAAATTGGCAAACACACAG & GACACAGCTTGAGAGAGAGAGAGA & 368 \\
rs1050171 & CGCATTCATGCGTCTTCACCTG & ATGGCAAACTCTTGCTATCCCA & \\
\hline
\end{tabular}

PCR, polymerase chain reaction; SNP, single-nucleotide polymorphism; EGF, growth factor receptor; EGFR, epidermal growth factor receptor.

rs11569017, Asp784Val) and 3 SNPs of the EGFR gene (rs6965469, -2004 C/T; rs2293347, Asp994Asp; and rs1050171, Gln787Gln). Additionally, the genotype of each SNP was determined through direct sequencing after polymerase chain reaction (PCR). PCR primers are shown in Table 2. Sequence data were analyzed using SeqManII software (v2.3; DNAATAR Inc., Madison, WI, USA).

\section{Statistical Analysis}

The derivation of tested SNPs in Hardy-Weinberg equilibrium was evaluated using SNPstats (http://bioinfo.iconcologia.net/ snpstats/start.htm). Differences in the genotypes and alleles of each SNP were analyzed by SNPstat and IBM SPSS Statistics ver. 20.0 (IBM Co., Armonk, NY, USA). The chi-square test and logistic regression with codominant, dominant, recessive, and logadditive models were utilized to analyze the association between tested polymorphisms and prostate volume, PSA level, or IPSS $[17,18]$. The linkage disequilibrium (LD) block and haplotypes between pairs of SNPs in each gene were tested using Haploview ver. 4.2 (Daly Laboratory, Cambridge, MA, USA). P-values $<0.05$ were considered to indicate statistical significance.

\section{RESULTS}

The demographic and biochemical characteristics of the participants are shown in Table 1. We analyzed the relationships between polymorphisms of the EGF and EGFR genes and BPH. The $\mathrm{BPH}$ patients were dichotomized according to prostate volume, IPSS score, and PSA level $[19,20]$. The genotype and allele distributions of the tested SNPs are shown in Tables 3 and 4 for each group. The derivation of the tested SNPs remained in Hardy-Weinberg equilibrium (rs3756261, P=0.35; rs11568835,
$\mathrm{P}=0.30 ;$ rs11568943, $\mathrm{P}=0.23 ; \mathrm{rs} 2237051, \mathrm{P}=0.51 ; \mathrm{rs} 11569017$, $\mathrm{P}=0.28$; and rs2293347, $\mathrm{P}=0.48$ in the $E G F$ gene; rs1050171, $\mathrm{P}=0.88$; and rs6965469, $\mathrm{P}=0.65$ in the EGFR gene).

First, we analyzed the relationship between polymorphisms of the EGF and EGFR genes and prostate volume. Codominant, dominant, recessive, and log-additive models were applied for statistical analysis. We found that EGF polymorphisms were associated with prostate volume in $\mathrm{BPH}$ patients (Table 3 ). The distributions of the $\mathrm{G}$ allele of rs11568943 and the A allele of rs11569017 in the EGF gene were significantly higher in patients with a prostate volume $\geq 30 \mathrm{~mL}$ than in patients with a prostate

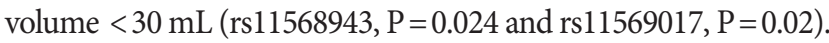
The genotype distributions of EGF polymorphisms also showed significant associations with prostate volume (rs11568943, $\mathrm{P}=0.038$ in the log-additive model [G/G vs. $A / G$ vs. $A / A]$; rs11569017, $\mathrm{P}=0.031$ in the dominant model [A/A vs. $\mathrm{A} / \mathrm{T}+\mathrm{T} / \mathrm{T}]$ and $\mathrm{P}=0.028$ in the log-additive model [A/A vs. $\mathrm{A} / \mathrm{T}$ vs. $\mathrm{T} / \mathrm{T}])$. In order to analyze haplotypes, we tested the LD block between paired SNPs in each gene. One LD block was found in the EGF gene among 3 SNPs (rs11568943, rs2237051, and rs11569017), and the LD block was strong (rs11568943 and rs2237051, D'=1.0, $r^{2}=0.109 ;$ rs11568943 and rs11569017, $D^{\prime}=0.985, r^{2}=0.812$; rs2237051 and rs11569017, $D^{\prime}=1.0, r 2=0.131$ ). Four haplotypes were found in the LD block of the EGF gene. Among these haplotypes, a significant difference were found according to AAT haplotype frequency in prostate volume $(<30 \mathrm{~mL}$ or $\geq 30 \mathrm{~mL})$ $(\mathrm{P}=0.017)$ (Table 5).

Second, we evaluated the relationship between polymorphisms of the EGF and EGFR genes and PSA levels, and found significant associations (Table 4). Three SNPs (rs3756261, -1744 A/G; rs11568943, Arg431Lys; and rs11569017, Asp784Val) in the EGF gene showed significant associations. The distributions 
Table 3. Genotype and allele distributions of tested SNPs in groups according to prostate volume

\begin{tabular}{|c|c|c|c|c|c|c|c|}
\hline \multirow{2}{*}{ SNP } & \multirow{2}{*}{$\begin{array}{c}\text { Genotype } \\
\text { Allele }\end{array}$} & \multicolumn{2}{|c|}{ Prostate volume (mL), n (\%) } & \multirow{2}{*}{ Model } & \multirow{2}{*}{ OR $(95 \% \mathrm{CI})$} & \multirow{2}{*}{ P-value } & \multirow{2}{*}{ P-value ${ }^{a)}$} \\
\hline & & $<30$ & $\geq 30$ & & & & \\
\hline \multirow{7}{*}{$\begin{array}{l}\text { EGF } \\
\text { rs3756261 } \\
-1744\end{array}$} & $\mathrm{~A} / \mathrm{A}$ & $49(50.5)$ & $75(62.0)$ & Codominant1 & $0.70(0.40-1.24)$ & 0.220 & \multirow{3}{*}{0.490} \\
\hline & $\mathrm{A} / \mathrm{G}$ & $43(44.3)$ & $42(34.7)$ & \multirow{6}{*}{$\begin{array}{l}\text { Codominant } 2 \\
\text { Dominant } \\
\text { Recessive } \\
\text { Log-additive }\end{array}$} & $0.55(0.14-2.18)$ & 0.400 & \\
\hline & G/G & $5(5.2)$ & $4(3.3)$ & & $0.68(0.39-1.19)$ & 0.180 & \\
\hline & & & & & $0.64(0.17-2.48)$ & 0.520 & \multirow[t]{4}{*}{0.520} \\
\hline & & & & & $0.72(0.45-1.15)$ & 0.170 & \\
\hline & A & $141(72.7)$ & $192(79.3)$ & & 1 & & \\
\hline & G & $53(27.3)$ & $50(20.7)$ & & $0.69(0.45-1.08)$ & 0.110 & \\
\hline \multirow{7}{*}{$\begin{array}{l}\text { EGF } \\
\text { rs11568835 } \\
-1380\end{array}$} & $\mathrm{G} / \mathrm{G}$ & $63(64.9)$ & $79(65.3)$ & Codominant1 & $1.01(0.56-1.85)$ & 0.960 & \multirow{7}{*}{$\begin{array}{l}0.550 \\
0.550\end{array}$} \\
\hline & G/A & $28(28.9)$ & $37(30.6)$ & Codominant 2 & $0.67(0.19-2.30)$ & 0.520 & \\
\hline & $\mathrm{A} / \mathrm{A}$ & $6(6.2)$ & $5(4.1)$ & Dominant & $0.95(0.54-1.68)$ & 0.870 & \\
\hline & & & & Recessive & $0.66(0.19-2.26)$ & 0.510 & \\
\hline & & & & Log-additive & $0.91(0.58-1.45)$ & 0.700 & \\
\hline & G & $154(79.4)$ & $195(80.6)$ & & 1 & & \\
\hline & $\mathrm{A}$ & $40(20.6)$ & $47(19.4)$ & & $0.93(0.58-1.49)$ & 0.760 & \\
\hline \multirow{7}{*}{$\begin{array}{l}\text { EGF } \\
\text { rs11568943 } \\
\text { Arg431Lys }\end{array}$} & $\mathrm{G} / \mathrm{G}$ & $50(51.5)$ & $80(66.1)$ & Codominant1 & $0.64(0.36-1.14)$ & 0.130 & \multirow{3}{*}{0.120} \\
\hline & $\mathrm{G} / \mathrm{A}$ & $42(43.3)$ & $39(32.2)$ & Codominant 2 & $0.25(0.05-1.37)$ & 0.110 & \\
\hline & $\mathrm{A} / \mathrm{A}$ & $5(5.2)$ & $2(1.6)$ & Dominant & $0.60(0.34-1.04)$ & 0.070 & \\
\hline & & & & Recessive & $0.30(0.06-1.60)$ & 0.140 & \multirow[t]{4}{*}{0.250} \\
\hline & & & & Log-additive & $0.60(0.36-0.98)$ & $0.038^{*}$ & \\
\hline & G & $142(73.2)$ & $199(82.2)$ & & 1 & & \\
\hline & A & $52(26.8)$ & $43(17.8)$ & & $0.59(0.37-0.93)$ & $0.024^{*}$ & \\
\hline & $\mathrm{A} / \mathrm{A}$ & $47(48.5)$ & $63(52.1)$ & Codominant1 & $0.68(0.39-1.20)$ & 0.190 & \\
\hline rs2237051 & $\mathrm{A} / \mathrm{G}$ & $47(48.5)$ & $46(38.0)$ & Codominant 2 & $2.93(0.77-11.09)$ & 0.110 & 0.160 \\
\hline Met708Ile & $\mathrm{G} / \mathrm{G}$ & $3(3.1)$ & $12(9.9)$ & Dominant & $0.82(0.47-1.41)$ & 0.460 & \\
\hline & & & & Recessive & $3.49(0.95-12.86)$ & 0.040 & 0.060 \\
\hline & & & & Log-additive & $1.05(0.68-1.63)$ & 0.830 & \\
\hline & A & $141(72.7)$ & $172(71.1)$ & & 1 & & \\
\hline & G & $53(27.3)$ & $70(28.9)$ & & $1.08(0.71-1.65)$ & 0.710 & \\
\hline EGF & $\mathrm{A} / \mathrm{A}$ & $44(45.4)$ & $75(62.0)$ & Codominant1 & $0.57(0.32-1.00)$ & 0.050 & \\
\hline rs11569017 & $\mathrm{A} / \mathrm{T}$ & $47(48.5)$ & $42(34.7)$ & Codominant2 & $0.40(0.11-1.50)$ & 0.170 & 0.180 \\
\hline Asp784Val & $\mathrm{T} / \mathrm{T}$ & $6(6.2)$ & $4(3.3)$ & Dominant & $0.55(0.32-0.95)$ & $0.031^{*}$ & \\
\hline & & & & Recessive & $0.51(0.14-1.88)$ & 0.300 & 0.350 \\
\hline & & & & Log-additive & $0.59(0.37-0.95)$ & $0.028^{*}$ & \\
\hline & A & $135(69.6)$ & $192(79.3)$ & & 1 & & \\
\hline & $\mathrm{T}$ & $59(30.4)$ & $50(20.7)$ & & $0.60(0.39-0.92)$ & $0.020^{*}$ & \\
\hline EGFR & $\mathrm{C} / \mathrm{C}$ & $63(64.9)$ & $84(69.4)$ & Codominant1 & $0.86(0.47-1.57)$ & 0.620 & \\
\hline rs6965469 & $\mathrm{C} / \mathrm{T}$ & $29(29.9)$ & $34(28.1)$ & Codominant2 & $0.43(0.10-1.88)$ & 0.260 & 0.300 \\
\hline-2004 & $\mathrm{~T} / \mathrm{T}$ & $5(5.2)$ & $3(2.5)$ & Dominant & $0.79(0.45-1.41)$ & 0.430 & \\
\hline & & & & Recessive & $0.45(0.10-1.95)$ & 0.280 & 0.470 \\
\hline & & & & Log-additive & $0.77(0.47-1.26)$ & 0.300 & \\
\hline & $\mathrm{C}$ & $155(79.9)$ & $202(83.5)$ & & 1 & & \\
\hline & $\mathrm{T}$ & $39(20.1)$ & $40(16.5)$ & & $0.79(0.48-1.28)$ & 0.340 & \\
\hline EGFR & $\mathrm{G} / \mathrm{G}$ & $44(45.4)$ & $59(48.8)$ & Codominant1 & $1.04(0.58-1.85)$ & 0.890 & \\
\hline rs2293347 & G/A & $41(42.3)$ & $52(42.9)$ & Codominant2 & $0.62(0.24-1.59)$ & 0.320 & \\
\hline Asp994Asp & $\mathrm{A} / \mathrm{A}$ & $12(12.4)$ & $10(8.3)$ & Dominant & $0.94(0.54-1.62)$ & 0.820 & \\
\hline & & & & Recessive & $0.61(0.25-1.50)$ & 0.280 & \\
\hline & & & & Log-additive & $0.87(0.58-1.31)$ & 0.510 & \\
\hline & G & $129(66.5)$ & $170(70.2)$ & & 1 & & \\
\hline & A & $65(33.5)$ & $72(29.8)$ & & $0.84(0.56-1.26)$ & 0.400 & \\
\hline EGFR & $\mathrm{G} / \mathrm{G}$ & $75(77.3)$ & $97(80.2)$ & Codominant1 & $0.91(0.47-1.78)$ & 0.800 & \\
\hline rs1050171 & $\mathrm{G} / \mathrm{A}$ & $21(21.6)$ & $24(19.8)$ & Codominant2 & $0.00(0.00-\mathrm{NA})$ & 1.000 & 0.440 \\
\hline Gln787Gln & $\mathrm{A} / \mathrm{A}$ & $1(1.1)$ & $0(0.0)$ & Dominant & $0.88(0.45-1.71)$ & 0.710 & \\
\hline & & & & Recessive & $0.00(0.00-\mathrm{NA})$ & 0.270 & 0.450 \\
\hline & & & & Log-additive & $0.85(0.45-1.61)$ & 0.610 & \\
\hline & G & $171(88.1)$ & $218(90.1)$ & & 1 & & \\
\hline & A & $23(11.9)$ & $24(9.9)$ & & $0.82(0.45-1.50)$ & 0.520 & \\
\hline
\end{tabular}

SNP, single nucleotide polymorphism; OR, odds ratio; CI, confidence interval; EGF, growth factor receptor; EGFR, epidermal growth factor receptor. ${ }^{\star} \mathrm{P}<0.05$, statistically significant. ${ }^{a)}$ Fisher exact test. 
Table 4. Genotype and allele distributions of tested SNPs in groups according to PSA level

\begin{tabular}{|c|c|c|c|c|c|c|c|}
\hline \multirow{2}{*}{ SNP } & \multirow{2}{*}{$\begin{array}{c}\text { Genotype } \\
\text { Allele }\end{array}$} & \multicolumn{2}{|c|}{ PSA (ng/mL), n (\%) } & \multirow{2}{*}{ Model } & \multirow{2}{*}{ OR $(95 \%$ CI $)$} & \multirow{2}{*}{ P-value } & \multirow{2}{*}{ P-value ${ }^{a)}$} \\
\hline & & $<1.5$ & $\geq 1.5$ & & & & \\
\hline \multirow{7}{*}{$\begin{array}{l}\text { EGF } \\
\text { rs3756261 } \\
-1744\end{array}$} & $\mathrm{~A} / \mathrm{A}$ & $33(44.0)$ & $91(64.1)$ & \multirow{7}{*}{$\begin{array}{l}\text { Codominant } 1 \\
\text { Codominant } 2 \\
\text { Dominant } \\
\text { Recessive } \\
\text { Log-additive }\end{array}$} & $0.48(0.26-0.87)$ & $0.016^{*}$ & \multirow[b]{2}{*}{0.260} \\
\hline & $\mathrm{A} / \mathrm{G}$ & $38(50.7)$ & $46(32.4)$ & & $0.48(0.12-1.91)$ & 0.300 & \\
\hline & $\mathrm{G} / \mathrm{G}$ & $4(5.3)$ & $5(3.5)$ & & $0.48(0.27-0.86)$ & $0.013^{*}$ & \multirow{5}{*}{0.500} \\
\hline & & & & & $0.66(0.17-2.56)$ & 0.550 & \\
\hline & & & & & $0.56(0.34-0.91)$ & $0.020^{*}$ & \\
\hline & A & $104(69.3)$ & $228(80.3)$ & & 1 & & \\
\hline & G & $46(30.7)$ & $56(19.7)$ & & $0.56(0.35-0.87)$ & $0.011^{*}$ & \\
\hline \multirow{6}{*}{$\begin{array}{l}\text { EGF } \\
\text { rs11568835 } \\
-1380\end{array}$} & $\mathrm{G} / \mathrm{G}$ & 47 (62.7) & $94(66.2)$ & \multirow{6}{*}{$\begin{array}{l}\text { Codominant1 } \\
\text { Codominant2 } \\
\text { Dominant } \\
\text { Recessive } \\
\text { Log-additive }\end{array}$} & $0.93(0.50-1.76)$ & 0.830 & \multirow{6}{*}{$\begin{array}{l}0.190 \\
0.200\end{array}$} \\
\hline & $\mathrm{G} / \mathrm{A}$ & $22(29.3)$ & $43(30.3)$ & & $0.41(0.12-1.43)$ & 0.160 & \\
\hline & $\mathrm{A} / \mathrm{A}$ & $6(8.0)$ & $5(3.5)$ & & $0.82(0.45-1.49)$ & 0.520 & \\
\hline & & & & & $0.42(0.12-1.44)$ & 0.170 & \\
\hline & G & $116(77.3)$ & $231(81.3)$ & & $\begin{array}{c}0.77(0.48-1.25) \\
\quad 1\end{array}$ & 0.290 & \\
\hline & A & $34(22.7)$ & $53(18.7)$ & & $0.78(0.48-1.27)$ & 0.320 & \\
\hline \multirow{6}{*}{$\begin{array}{l}\text { EGF } \\
\text { rs11568943 } \\
\text { Arg431Lys }\end{array}$} & $\mathrm{G} / \mathrm{G}$ & $36(48.0)$ & $94(66.2)$ & \multirow{6}{*}{$\begin{array}{l}\text { Codominant1 } \\
\text { Codominant } 2 \\
\text { Dominant } \\
\text { Recessive } \\
\text { Log-additive }\end{array}$} & $0.52(0.29-0.94)$ & $0.031^{*}$ & \multirow{6}{*}{$\begin{array}{l}0.410 \\
0.700\end{array}$} \\
\hline & $\mathrm{G} / \mathrm{A}$ & $36(48.0)$ & $44(31.0)$ & & $0.52(0.11-2.46)$ & 0.410 & \\
\hline & $\mathrm{A} / \mathrm{A}$ & $3(4.0)$ & $4(2.8)$ & & $0.52(0.29-0.93)$ & $0.027^{*}$ & \\
\hline & & & & & $0.68(0.15-3.15)$ & 0.620 & \\
\hline & G & $108(72.0)$ & $232(81.7)$ & & $0.58(0.35-0.97)$ & $0.036^{*}$ & \\
\hline & A & $42(28.0)$ & $52(18.3)$ & & $0.58(0.36-0.92)$ & $0.021^{*}$ & \\
\hline \multirow{6}{*}{$\begin{array}{l}\text { EGF } \\
\text { rs2237051 } \\
\text { Met708Ile }\end{array}$} & $\mathrm{A} / \mathrm{A}$ & $40(53.3)$ & $70(49.3)$ & \multirow{6}{*}{$\begin{array}{l}\text { Codominant1 } \\
\text { Codominant2 } \\
\text { Dominant } \\
\text { Recessive } \\
\text { Log-additive }\end{array}$} & $1.01(0.56-1.81)$ & 0.980 & \\
\hline & $\mathrm{A} / \mathrm{G}$ & $32(42.7)$ & $60(42.2)$ & & $2.23(0.59-8.46)$ & 0.240 & \\
\hline & $\mathrm{G} / \mathrm{G}$ & $3(4.0)$ & $12(8.4)$ & & $1.11(0.63-1.96)$ & 0.720 & 50 \\
\hline & & & & & $2.22(0.60-8.21)$ & 0.200 & 0.270 \\
\hline & A & $112(74.7)$ & $200(70.4)$ & & $1.21(0.76-1.92)$ & 0.430 & \\
\hline & G & $38(25.3)$ & $84(29.6)$ & & $1.24(0.79-1.94)$ & 0.350 & \\
\hline EGF & $\mathrm{A} / \mathrm{A}$ & $33(44.0)$ & $86(60.6)$ & Codominant1 & $0.55(0.30-0.99)$ & 0.050 & \\
\hline rs11569017 & $\mathrm{A} / \mathrm{T}$ & $38(50.7)$ & $50(35.2)$ & Codominant 2 & $0.59(0.15-2.24)$ & 0.440 & 0.470 \\
\hline Asp784Val & $\mathrm{T} / \mathrm{T}$ & $4(5.3)$ & $6(4.2)$ & Dominant & $0.55(0.31-0.99)$ & $0.044^{*}$ & \\
\hline & & & & Recessive & $0.77(0.21-2.85)$ & 0.700 & 0.740 \\
\hline & A & $104(693)$ & $222(782)$ & Log-additive & $0.64(0.39-1.03)$ & 0.070 & \\
\hline & $\mathrm{T}$ & $46(30.7)$ & $62(21.8)$ & & ${ }_{0.63}^{1}(0.40-0.99)$ & $0.044^{*}$ & \\
\hline EGFR & $\mathrm{C} / \mathrm{C}$ & 47 (62.7) & $100(70.4)$ & Codominant1 & $0.77(0.41-1.46)$ & 0.430 & \\
\hline rs6965469 & $\mathrm{C} / \mathrm{T}$ & $23(30.7)$ & $39(27.5)$ & Codominant 2 & $0.27(0.06-1.16)$ & 0.080 & 0.120 \\
\hline-2004 & $\mathrm{~T} / \mathrm{T}$ & $5(6.7)$ & $3(2.1)$ & Dominant & $0.68(0.37-1.24)$ & 0.210 & \\
\hline & & & & Recessive & $0.29(0.07-1.24)$ & 0.090 & 0.130 \\
\hline & $\mathrm{C}$ & $117(78.0)$ & $239(84.2)$ & Log-additive & $0.65(0.39-1.08)$ & 0.100 & \\
\hline & $\mathrm{T}$ & $33(22.0)$ & $45(15.8)$ & & $0.67(0.41-1.10)$ & 0.110 & \\
\hline EGFR & $\mathrm{G} / \mathrm{G}$ & $41(54.7)$ & $61(42.9)$ & Codominant1 & $1.61(0.88-2.96)$ & 0.120 & \\
\hline rs2293347 & $\mathrm{G} / \mathrm{A}$ & $30(40.0)$ & $63(44.4)$ & Codominant 2 & $3.20(0.99-10.35)$ & 0.050 & 0.060 \\
\hline Asp994Asp & $\mathrm{A} / \mathrm{A}$ & $4(5.3)$ & $18(12.7)$ & Dominant & $1.81(1.01-3.23)$ & $0.044^{*}$ & \\
\hline & & & & Recessive & $2.56(0.82-7.98)$ & 0.080 & 0.100 \\
\hline & & & & Log-additive & $1.70(1.07-2.71)$ & 0.020 & \\
\hline & $\begin{array}{l}\mathrm{G} \\
\mathrm{A}\end{array}$ & $\begin{array}{r}112(74.7) \\
38(25.3)\end{array}$ & $\begin{array}{r}185(65.1) \\
99(34.9)\end{array}$ & & $\begin{array}{c}1 \\
158(101-245)\end{array}$ & $0043 *$ & \\
\hline EGFR & $\mathrm{G} / \mathrm{G}$ & $55(73.3)$ & $116(81.7)$ & Codominant1 & $0.67(0.34-1.32)$ & 0.250 & \\
\hline rs1050171 & $\mathrm{G} / \mathrm{A}$ & $19(25.3)$ & $26(18.3)$ & Codominant 2 & $0.00(0.00-\mathrm{NA})$ & 1.000 & 0.330 \\
\hline Gln787Gln & $\mathrm{A} / \mathrm{A}$ & $1(1.4)$ & $0(0.0)$ & Dominant & $0.64(0.33-1.26)$ & 0.200 & \\
\hline & & & & Recessive & $0.00(0.00-\mathrm{NA})$ & 0.200 & 0.360 \\
\hline & & & & Log-additive & $0.62(0.32-1.19)$ & 0.150 & \\
\hline & G & $129(86.0)$ & $258(90.8)$ & & 1 & & \\
\hline & A & $21(14.0)$ & $26(9.2)$ & & $0.62(0.34-1.14)$ & 0.130 & \\
\hline
\end{tabular}

SNP, single nucleotide polymorphism; PSA, prostate-specific antigen; OR, odds ratio; CI, confidence interval; EGF, growth factor receptor; EGFR, epidermal growth factor receptor.

${ }^{*} \mathrm{P}<0.05$, statistically significant. ${ }^{\text {a) }}$ Fisher exact test. 
Table 5. Haplotype analysis in rs11568943, rs2237051, and rs11569017 of EGF gene

\begin{tabular}{|c|c|c|c|c|c|c|c|}
\hline \multirow{2}{*}{ Haplotype } & \multirow{2}{*}{ Frequency } & \multicolumn{2}{|c|}{ Group 1} & \multicolumn{2}{|c|}{ Group 2} & \multirow{2}{*}{ Chi-square } & \multirow{2}{*}{ P-value } \\
\hline & & + & - & + & - & & \\
\hline & & \multicolumn{2}{|c|}{ Prostate volume $(<30 \mathrm{~mL})$} & \multicolumn{2}{|c|}{ Prostate volume ( $\geq 30 \mathrm{~mL})$} & & \\
\hline GAA & 0.468 & 82 & 112 & 122 & 120 & 2.869 & 0.090 \\
\hline GGA & 0.280 & 53 & 141 & 69 & 173 & 0.077 & 0.780 \\
\hline AAT & 0.215 & 52 & 142 & 42 & 200 & 5.679 & $0.017^{*}$ \\
\hline \multirow[t]{2}{*}{ GAT } & 0.035 & 7 & 187 & 8 & 234 & 0.031 & 0.860 \\
\hline & & \multicolumn{2}{|c|}{ PSA $(<1.5 \mathrm{ng} / \mathrm{mL})$} & \multicolumn{2}{|c|}{$\mathrm{PSA}(\geq 1.5 \mathrm{ng} / \mathrm{mL})$} & & \\
\hline GAA & 0.470 & 66 & 84 & 138 & 146 & 0.831 & 0.360 \\
\hline GGA & 0.279 & 38 & 112 & 83 & 201 & 0.741 & 0.390 \\
\hline AAT & 0.214 & 42 & 108 & 51 & 233 & 5.876 & $0.015^{*}$ \\
\hline GAT & 0.035 & 4 & 146 & 11 & 273 & 0.424 & 0.520 \\
\hline
\end{tabular}

PSA, prostate-specific antigen.

${ }^{*} \mathrm{P}<0.05$, statistically significant.

of the major alleles of rs3756261 and rs11568943 were higher in patients with a PSA level $\geq 1.5 \mathrm{ng} / \mathrm{mL}$ than in patients with PSA levels $<1.5 \mathrm{ng} / \mathrm{mL}$ (rs3756261, $\mathrm{P}=0.011$; rs11568943, $\mathrm{P}=0.021$ ). The genotype distributions of $E G F$ polymorphisms also displayed significant differences ( $r s 3756261, \mathrm{P}=0.016$ in the codominant 1 model [A/A vs. A/G], P=0.013 in the dominant model $[\mathrm{A} / \mathrm{A}$ vs. $\mathrm{A} / \mathrm{G}+\mathrm{G} / \mathrm{G}], \mathrm{P}=0.020$ in the log-additive model $[A / A$ vs. $A / G$ vs. $G / G]$; rs11568943, $P=0.031$ in the codominant 1 model [G/G vs. G/A], $P=0.027$ in the dominant model $[\mathrm{G} / \mathrm{G}$ vs. $\mathrm{G} / \mathrm{A}+\mathrm{A} / \mathrm{A}], \mathrm{P}=0.036$ in the log-additive model [G/G vs. $G / A$ vs. $A / A]$; rs11569017, $P=0.044$ in the dominant model [A/A vs. A/T+T/T]). Additionally, rs2293347 in the EGFR gene showed a relationship with PSA $(\mathrm{P}=0.044$ in the dominant model [G/G vs. $G / A+A / A], P=0.020$ in the log-additive model $[G / G$ vs. $G / A$ vs. $A / A]$, and $P=0.043$ in the allele distribution]. In the haplotype analysis, a significant association was found between the AAT haplotype in the EGF gene and PSA levels $(P=0.015)$ (Table 5).

However, we did not find any significant associations between polymorphisms of the EGF and EGFR genes and IPSS.

\section{DISCUSSION}

The pathogenesis of BPH is still unknown. However, several studies have reported that specific polymorphisms in various genes contribute to the pathogenesis of $\mathrm{BPH}$ [21]. The most representative genes of this type is the androgen receptor $(A R)$ gene. $\mathrm{AR}$ is a transactivation factor that depends on the binding of steroid hormones. It has an important role in the proliferation and differentiation of prostate cells [22]. Polymorphic variations are present in the $A R$ gene. It has been suggested that the presence of a higher number of polymorphic GGC repeats in the $A R$ gene is associated with an increased risk of developing $\mathrm{BPH}$ [23]. Short CAG alleles may be a genetic factor that promotes the growth of $\mathrm{BPH}$ [24].

The relationship between EGF and EGFR polymorphisms and the clinical features of BPH has not been previously investigated. In the current study, associations between SNPs in the $E G F$ and EGFR genes and BPH were evaluated. The prostate volume of $\mathrm{BPH}$ patients was associated with the EGF SNPs rs11568943 and rs11569017, and PSA levels in BPH patients were associated with the EGF SNPs rs11568943, rs11569017, and rs3756261 and the EGFR SNP rs2293347.

In previous studies of the $E G F$ and $E G F R$ genes, the rs 11568943 SNP was significantly associated with preeclampsia [25], psoriatic arthritis [26], and gastric cancer [27]. The rs 11569017 SNP was associated with the risk of hepatitis B virus-related hepatocellular carcinoma [28]. The rs3756261 SNP was also showed a significant association with a higher risk of developing preeclampsia [25]. Among the EGFR SNPs, the rs2293347 SNP is associated with chemotherapeutic response, lung cancer treated with gefitinib, and airway hyperresponsiveness.

EGF binding induces the dimerization of EGFR and the activation of downstream signaling pathways involved in regulating cellular proliferation, differentiation, and survival [29]. This EGF-EGFR ligand-receptor complex stimulates cell prolifera- 
tion [30]. EGF protects epithelial cells against Fas-induced apoptosis [31], and EGFR plays an essential role in the morphogenesis of mammary glands [32]. EGF controls myoepithelial cell differentiation in mammary gland cultures [33] and EGFR is closely linked to smooth cell apoptosis [34]. The prostate gland consists of the peripheral zone, central zone, and transition zone. The transition zone, which contains smooth muscle cells and myoepithelial cells, is responsible for BPH [35]. As described above, EGF and EGFR may be related to various aspects of epithelial and muscular proliferation, which could affect BPH.

In summary, no associations between the EGF and EGFR genes and the clinical features of BPH have previously been reported. We found for the first time that 2 SNPs (rs11568943 and rs11569017) and the AAT haplotype in the EGF gene may affect prostate volume, and that 3 SNPs (rs11568943, rs11569017, and rs3756261) and the AAT haplotype in EGF gene, as well as 1 SNP in the EGFR gene (rs2293347), may affect PSA levels in $\mathrm{BPH}$ patients. However, this study has limitations such as sample size, ethnic differences, lack of control subjects, and interaction with environmental factors. To confirm our results, a casecontrol study in another population with a larger sample size is needed, as well as a meta-analysis.

\section{REFERENCES}

1. Thapa D, Ghosh R. Chronic inflammatory mediators enhance prostate cancer development and progression. Biochem Pharmacol 2015;94:53-62.

2. Damodarasamy M, Vernon RB, Chan CK, Plymate SR, Wight TN, Reed MJ. Hyaluronan in aged collagen matrix increases prostate epithelial cell proliferation. In Vitro Cell Dev Biol Anim 2015;51: 50-8.

3. Soulitzis N, Karyotis I, Delakas D, Spandidos DA. Expression analysis of peptide growth factors VEGF, FGF2, TGFB1, EGF and IGF1 in prostate cancer and benign prostatic hyperplasia. Int J Oncol 2006;29:305-14.

4. Kim HG, Kassis J, Souto JC, Turner T, Wells A. EGF receptor signaling in prostate morphogenesis and tumorigenesis. Histol Histopathol 1999;14:1175-82.

5. Pollan MC, Benghuzzi HA, Tucci M. Growth factor expression in early stages of benign prostatic hyperplasia upon exposure to sustained delivery of androgens. Biomed Sci Instrum 2003;39:329-34.

6. Monti S, Sciarra F, Adamo MV, Toscano V, Trotta MC, Martini C, et al. Prevalent decrease of the EGF content in the periurethral zone of BPH tissue induced by treatment with finasteride or flu- tamide. J Androl 1997;18:488-94.

7. Davies P, Eaton CL, France TD, Phillips ME. Growth factor receptors and oncogene expression in prostate cells. Am J Clin Oncol 1988;11 Suppl 2:S1-7.

8. Grabowska MM, Sandhu B, Day ML. EGF promotes the shedding of soluble E-cadherin in an ADAM10-dependent manner in prostate epithelial cells. Cell Signal 2012;24:532-8.

9. Maslova K, Kyriakakis E, Pfaff D, Frachet A, Frismantiene A, Bubendorf L, et al. EGFR and IGF-1R in regulation of prostate cancer cell phenotype and polarity: opposing functions and modulation by T-cadherin. FASEB J 2015;29:494-507.

10. Teixeira AL, Ribeiro R, Morais A, Lobo F, Fraga A, Pina F, et al. Combined analysis of EGF $+61 \mathrm{G}>\mathrm{A}$ and TGFB1 $+869 \mathrm{~T}>\mathrm{C}$ functional polymorphisms in the time to androgen independence and prostate cancer susceptibility. Pharmacogenomics J 2009;9:341-6.

11. Teixeira AL, Ribeiro R, Cardoso D, Pinto D, Lobo F, Fraga A, et al. Genetic polymorphism in EGF is associated with prostate cancer aggressiveness and progression-free interval in androgen blockadetreated patients. Clin Cancer Res 2008;14:3367-71.

12. Fu M, Zhang W, Shan L, Song J, Shang D, Ying J, et al. Mutation status of somatic EGFR and KRAS genes in Chinese patients with prostate cancer (PCa). Virchows Arch 2014;464:575-81.

13. de Muga S, Hernández S, Agell L, Salido M, Juanpere N, Lorenzo $\mathrm{M}$, et al. Molecular alterations of EGFR and PTEN in prostate cancer: association with high-grade and advanced-stage carcinomas. Mod Pathol 2010;23:703-12.

14. Chen Y, Xin X, Li J, Xu J, Yu X, Li T, et al. RTK/ERK pathway under natural selection associated with prostate cancer. PLoS One 2013;8: e78254.

15. Lin HY, Amankwah EK, Tseng TS, Qu X, Chen DT, Park JY. SNPSNP interaction network in angiogenesis genes associated with prostate cancer aggressiveness. PLoS One 2013;8:e59688.

16. Perez CA, Chen H, Shyr Y, Courtney R, Zheng W, Cai Q, et al. The EGFR polymorphism rs884419 is associated with freedom from recurrence in patients with resected prostate cancer. J Urol 2010; 183:2062-9.

17. Kim HK, Lee H, Kim HJ. A polymorphism in DMT1 is associated with lead-related hypertensive status. Mol Cell Toxicol 2013;9:41520.

18. Park HK, Kim SK. Promoter polymorphisms of NDUFA4 gene were associated with prostate enlargement of benign prostatic hyperplasia. Mol Cell Toxicol 2015;11:401-6.

19. Atan A, Güzel Ö. How should prostate specific antigen be interpreted? Turk J Urol 2013;39:188-93.

20. Crawford ED, Wilson SS, McConnell JD, Slawin KM, Lieber MC, 
Smith JA, et al. Baseline factors as predictors of clinical progression of benign prostatic hyperplasia in men treated with placebo. J Urol 2006;175:1422-6.

21. Konwar R, Chattopadhyay N, Bid HK. Genetic polymorphism and pathogenesis of benign prostatic hyperplasia. BJU Int 2008;102: 536-44.

22. Chamberlain NL, Driver ED, Miesfeld RL. The length and location of CAG trinucleotide repeats in the androgen receptor $\mathrm{N}$-terminal domain affect transactivation function. Nucleic Acids Res 1994;22: 3181-6.

23. Biolchi V, Silva Neto B, Pianta DB, Koff WJ, Berger M, Brum IS. Androgen receptor GGC polymorphism and testosterone levels associated with high risk of prostate cancer and benign prostatic hyperplasia. Mol Biol Rep 2013;40:2749-56.

24. Mitsumori K, Terai A, Oka H, Segawa T, Ogura K, Yoshida O, et al. Androgen receptor $\mathrm{CAG}$ repeat length polymorphism in benign prostatic hyperplasia (BPH): correlation with adenoma growth. Prostate 1999;41:253-7.

25. Chenthuran T, Galhenagey GH, Jayasekara RW, Dissanayake VH. Polymorphism in the epidermal growth factor gene is associated with pre-eclampsia and low birthweight. J Obstet Gynaecol Res 2014;40:1235-42.

26. Butt C, Lim S, Greenwood C, Rahman P. VEGF, FGF1, FGF2 and EGF gene polymorphisms and psoriatic arthritis. BMC Musculoskelet Disord 2007;8:1.

27. Zhan Z, Chen Y, Wu J, Zhang J, Tong S, Zhang C, et al. Functional epidermal growth factor gene polymorphisms and risk of gastric cancer. Oncol Lett 2013;5:631-6.

28. Wu J, Zhang W, Xu A, Zhang L, Yan T, Li Z, et al. Association of epidermal growth factor and epidermal growth factor receptor polymorphisms with the risk of hepatitis B virus-related hepatocellular carcinoma in the population of North China. Genet Test Mol Biomarkers 2013;17:595-600.

29. Herbst RS. Review of epidermal growth factor receptor biology. Int J Radiat Oncol Biol Phys 2004;59(2 Suppl):21-6.

30. Pennock S, Wang Z. Stimulation of cell proliferation by endosomal epidermal growth factor receptor as revealed through two distinct phases of signaling. Mol Cell Biol 2003;23:5803-15.

31. Gibson S, Tu S, Oyer R, Anderson SM, Johnson GL. Epidermal growth factor protects epithelial cells against Fas-induced apoptosis. Requirement for Akt activation. J Biol Chem 1999;274:17612-8.

32. Sebastian J, Richards RG, Walker MP, Wiesen JF, Werb Z, Derynck $\mathrm{R}$, et al. Activation and function of the epidermal growth factor receptor and erbB-2 during mammary gland morphogenesis. Cell Growth Differ 1998;9:777-85.

33. Petersen OW, van Deurs B. Growth factor control of myoepithelialcell differentiation in cultures of human mammary gland. Differentiation 1988;39:197-215.

34. Merklinger SL, Jones PL, Martinez EC, Rabinovitch M. Epidermal growth factor receptor blockade mediates smooth muscle cell apoptosis and improves survival in rats with pulmonary hypertension. Circulation 2005;112:423-31.

35. McNeal JE. Normal histology of the prostate. Am J Surg Pathol 1988;12:619-33. 